\title{
Simultaneous two-layer harvesting of scalp split- thickness skin and dermal grafts for acute burns and postburn scar deformities
}

\author{
Suk Joon Oh \\ Department of Burn Reconstructive Surgery, Bestian Seoul Hospital, Seoul, Korea
}

Background The scalp, an excellent donor site for thin skin grafts, presents a limited surface but is rich in stem cells. The purpose of this study was to test a double harvesting procedure from the scalp and to evaluate the capacity of the dermal layer.

Methods Two layers corresponding to a split-thickness skin graft (SSG) and a split-thickness dermal graft (SDG) were harvested from the scalp using a Zimmer dermatome during the same procedure. Healing of the scalp donor site, reason for recipient site grafting, and the percentage of graft loss were evaluated.

Results Fourteen patients, comprising six men and eight women with a mean age of 34.2 years, were treated according to our protocol. The most common reason for a recipient site graft was a postburn scar deformity (10/14 patients). The mean area of scalp SSGs was 151.8 $\mathrm{cm}^{2}$. The mean area of scalp SDGs was $88.2 \mathrm{~cm}^{2}$. The mean healing time of scalp donors was 9.9 days. The only donor complication was a tufted scar deformity.

Conclusions Skin defects in the scalp of donors healed faster and led to less scarring than defects at other donor sites. Scalp SDGs needed 10 days for adequate epithelization. The scalp was the best donor site for SSGs and SDGs for burn reconstructive patients.

Keywords Scalp / Grafts / Wound healing / Regeneration
Correspondence: Suk Joon Oh Department of Burn Reconstructive Surgery, Bestian Seoul Hospital, 429 Dogok-ro, Gangnam-gu, Seoul 06208, Korea

Tel: +82-70-7609-9321

Fax: $+82-70-7005-4233$

E-mail:sjoh46@nate.com

Received: August 17, 2018 • Revised: October 25, 2018 • Accepted: June 27, 2019

pISSN: 2234-6163 • elSSN: 2234-6171 • https://doi.org/10.5999/aps.2018.00990 • Arch Plast Surg 2019;46:558-565

\section{INTRODUCTION}

Crawford (1964) [1] first introduced the scalp as an unusual skin donor site in the treatment of extensive deep burns in children. The scalp, although it is hairy, is a highly favorable donor site for obtaining thin skin grafts for burn patients [2-5]. Scalp donor sites heal rapidly because they are rich in hair follicle structures, which contain abundant regenerative epithelial cells and dermis cells. Another major advantage of the scalp as a donor site is that scarring is not visible. However, the surface of the scalp makes up a limited proportion of the entire body surface.

To overcome the disadvantage of the relatively small area of the scalp as donor, Zakine et al. [6] reported harvesting two layers of a 0.2 -mm graft, a dermoepidermal graft and a dermal graft, from the scalp during a single procedure in 15 burn patients. Scalp dermal grafts contain abundant epithelial and regenerative dermal cells, which facilitate complete healing of the recipient site. Dermal autografts from the scalp have been mainly used in burned wounds, although they can also be used in various other types of wounds. However, there are no reports 
that used scalp split-thickness skin graft (SSG) and split-thickness dermal graft (SDG) for postburn scar contractures (PB$\mathrm{SCs}$ ) and postburn scar deformities (PBSDs) of the face or lower extremities.

This study aimed to double the scalp donor surface by harvesting a thin SDG immediately after a thin SSG. We also verified the quality of recipient site healing after receiving a SSG and a SDG and scalp healing after double graft harvesting in burn and reconstructive patients.

\section{METHODS}

Fourteen Korean patients hospitalized in our burn and reconstructive unit were included in this retrospective study between August 2013 and July 2017. Data on factors including sex, age, reason for surgery, recipient site, graft thickness, and area of each scalp SSG and SDG at the time of surgery were collected from the medical records of the included patients. The postoperative variables included the healing time of the donor site, epithelialization time of the scalp SDG, and incidence of complications. After follow-up for 1 year, the incidence of late complications and the Vancouver Scar Scale scores of the grafts were reviewed.

\section{Surgical technique}

The recipient sites for grafting were prepared by debriding burn wounds and flap donor wounds, shaving the scars of dyspigmented and uneven PBSDs, and releasing PBSCs covered with artificial dermal matrix $(\mathrm{ADM})$.

The scalp was shaved and sterilized with a povidone solution containing $70 \%$ alcohol. Then, 1:300,000 adrenaline mixed with sterile physiologic saline $(\mathrm{NaCl} 0.9 \%)$ was infiltrated into the subgaleal space to create a cushion in order to harvest a broad re- gion of skin. The dual scalp SSG and SDG were then harvested with a Zimmer Air Dermatome (Zimmer Inc., Warsaw, IN, USA). Hemostasis was achieved by using a temporary gauze dressing soaked in adrenaline solution containing $10 \mathrm{mg}$ adrenaline in $1 \mathrm{~L} \mathrm{NaCl}$ 0.9\%. After hemostasis, a Mepitel sheet (Mölnlycke Health Care, Gothenburg, Sweden) was tightly fixed with staples to the donor site, covered with absorbent gauze and secured with an elastic bandage and an elastic stocking.

Exudates were absorbed by the gauze on the Mepitel sheet, which was changed at the scalp donor site between 3 and 5 days after surgery. After this period, the epithelialization of scalp wounds was observed beneath the Mepitel sheet after partially removing the staples (Fig. 1). The upper bandages, absorbent gauze and Mepitel sheet can be removed from the donor site

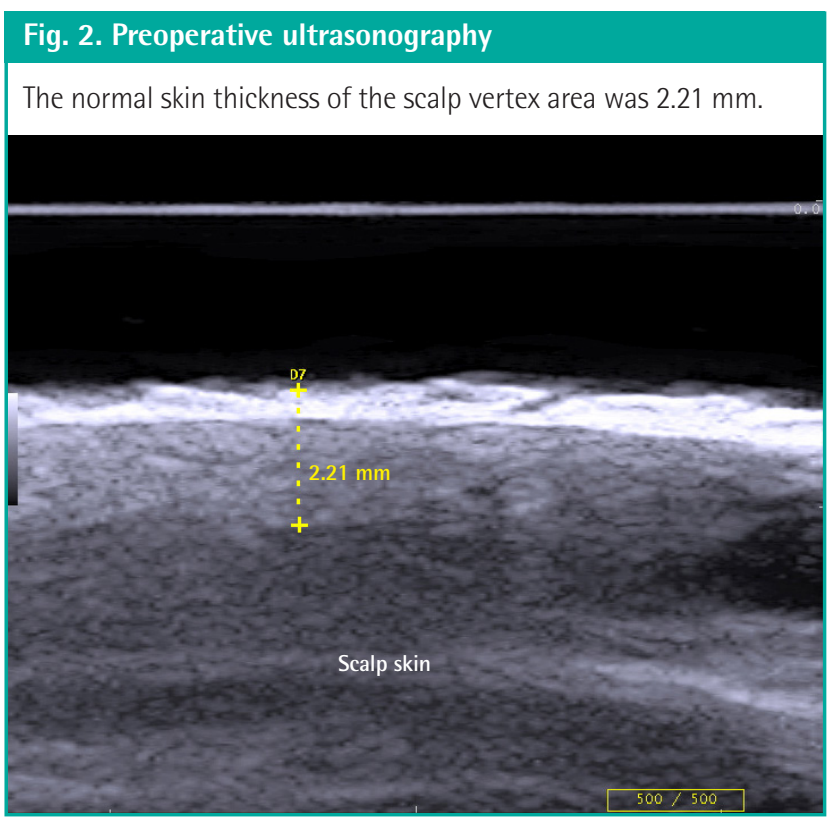

\section{Fig. 1. Photographic findings of scalp donor healing}

(A) Whitish areas of epidermis regeneration around the hairs were observed at 3 days postoperatively. (B) Complete healing of the scalp donor wound was observed at 9 days postoperatively.
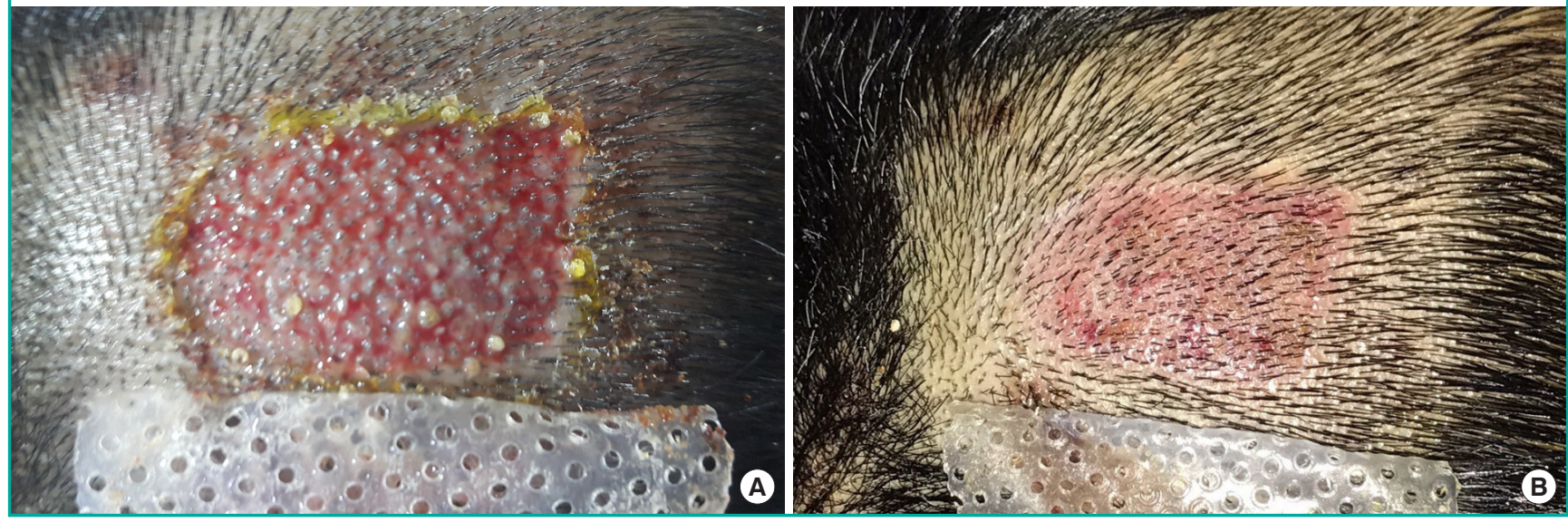
when the site is fully epithelized.

After grafting, graft maturation of the scalp SSG and SDG recipient areas was managed for up to one year with moisturizing cream, silicone gel or pad and pressure garments $(20-25 \mathrm{mmHg}$ pressure).

\section{Assessment}

The scalp skin thickness was measured preoperatively by ultrasonography (Esaote MyLab One, Genoa, Italy) (Fig. 2). Ultrasonography measured the skin thickness after shaving the hair on the temporal, vertex, parieto-temporal and occipital areas in patients requiring large-sized skin grafts, and on the temporooccipital area in patients requiring small-sized skin grafts. We confirmed the histology of the scalp SSG and SDGs (Fig. 3). Scalp donor healing was assessed by histological findings of punch biopsy (Fig. 4). Donor site healing was defined as greater than $95 \%$ epithelialization of the donor site.

Postoperative variables were healing time of the donor site, epithelialization time of scalp SDG and incidence of complications. After follow-up for one year, the incidence of late compli-

\section{Fig. 3. Histology of the scalp SSG and SDG}

Histological findings $\left(H \& E_{1} \times 100\right)$. (A) SSG with epidermis and papillary dermis. (B) SDG with reticular dermis, including adnexal structures rich in keratinocytes. SSG, split-thickness skin graft; SDG, split-thickness dermal graft.
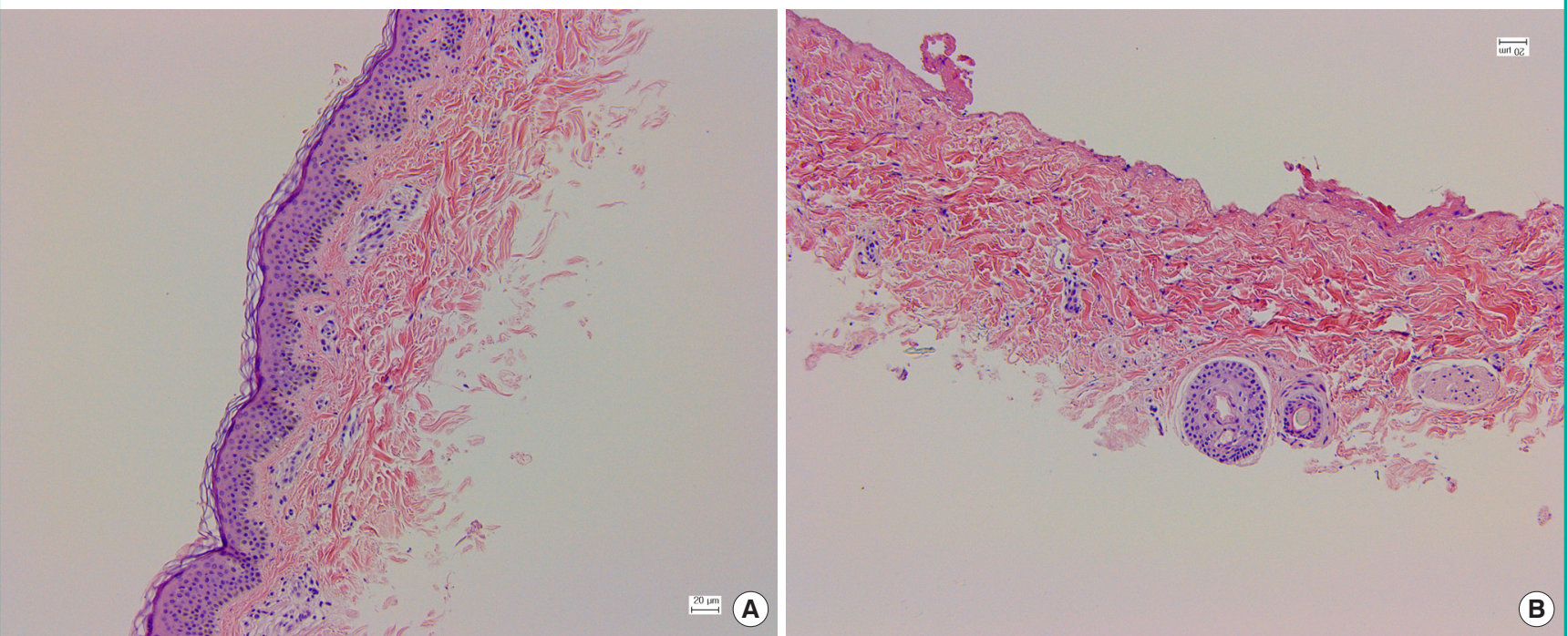

Fig. 4. Histological findings of the scalp donor site

Histological findings of the scalp donor site at 9 days postoperatively. (A) Regenerated epithelium and regenerated dermal fibroblasts (H\&E, $\times 40)$. (B) Regenerated dermal fibroblasts from the deep reticular border were stained dark blue according to the degree of differentiated collagen formation (Masson trichrome stain, $\times 40$ ).



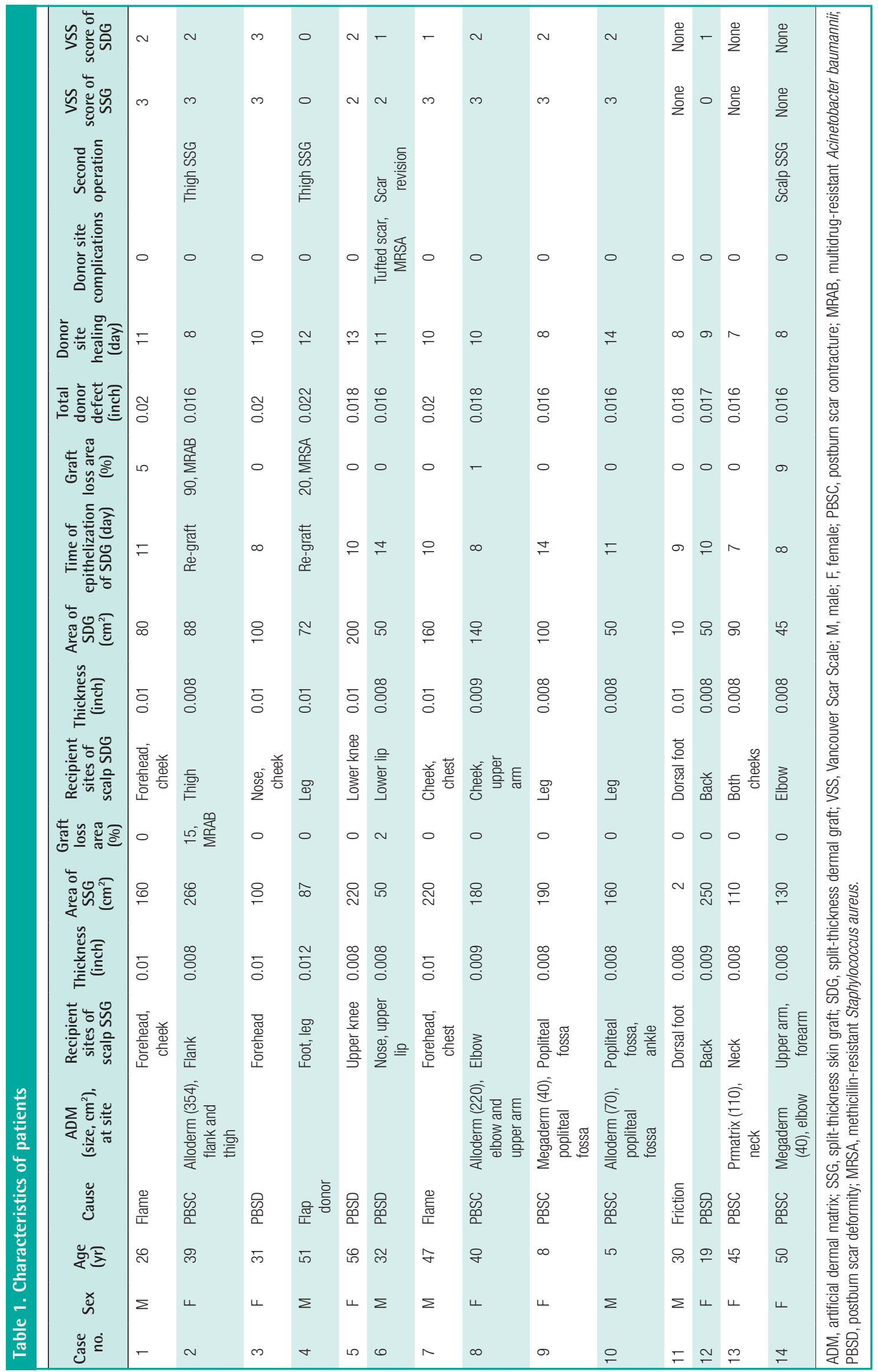


cations and the Vancouver scar scale scores of the grafts were reviewed.

\section{RESULTS}

Fourteen patients, six men and eight women with a mean age of
34.2 years (range, 5-56 years), were treated according to our protocol. The reasons for recipient site grafting were PBSCs (6 patients), PBSDs (4 patients), flame burns (2 patients), flap donor site ( 1 patient), and friction wound ( 1 patient) (Table 1 ).

The recipient sites of scalp SSGs were the face $(n=5)$, lower extremity $(n=5)$, body $(n=2)$ and upper extremity $(n=2)$, and

\section{Fig. 5. Scalp SSG and SDG in case 8}

Excision release of postburn scar contracture, Alloderm graft, and scalp split-thickness dermal graft (SDG) and split-thickness skin graft (SSG). (A) Preoperative postburn scar contracture around the left elbow. (B) An Alloderm graft covered the wound. (C) A scalp SDG was placed over the upper part of the Alloderm graft wound and a scalp SSG over the lower part. (D) Incomplete maturation of the grafts at 1 year and 8 months postoperatively.
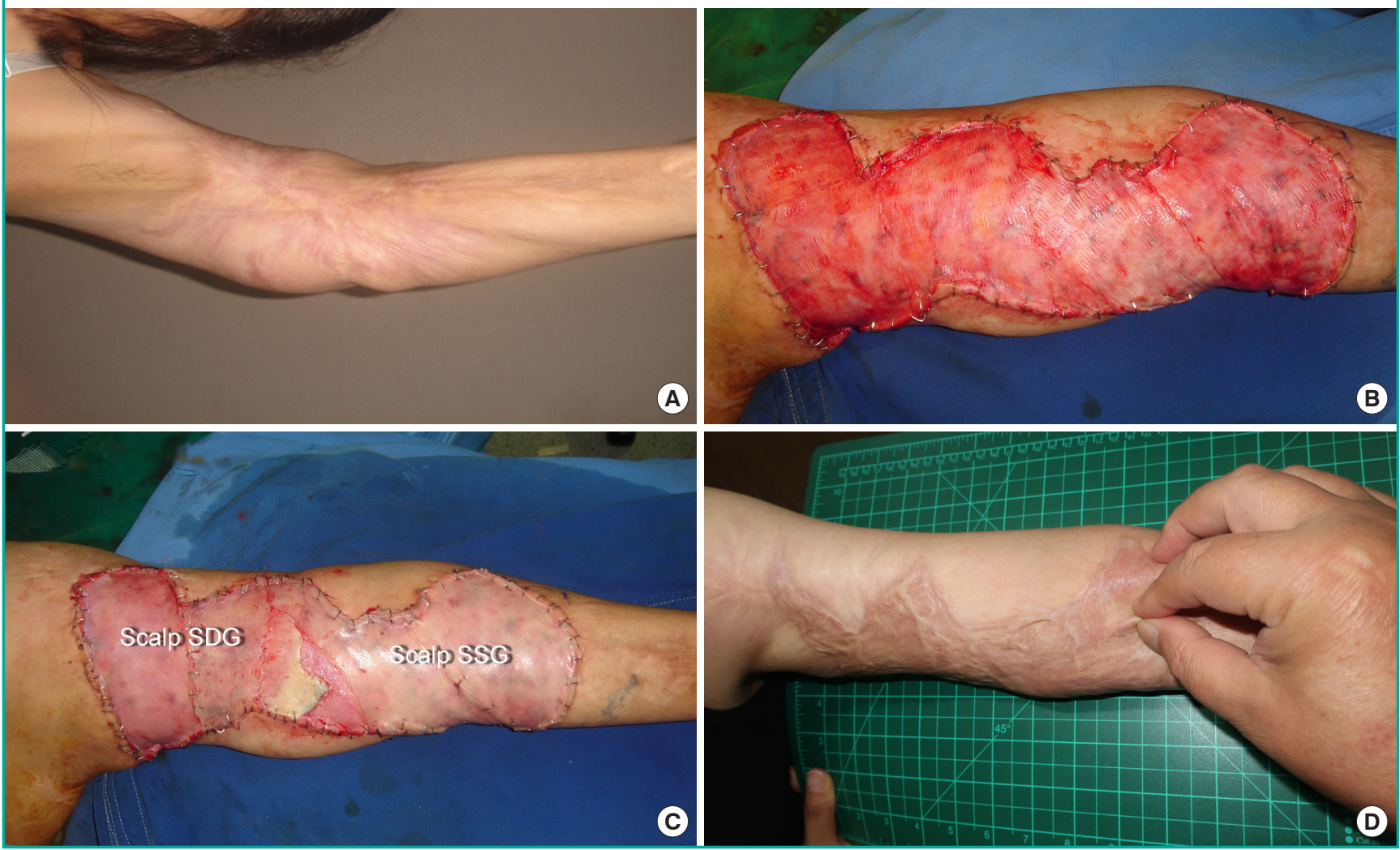

Fig. 6. Scalp SSG and SDG in case 7

Debridement and scalp split-thickness skin graft (SSG) and split-thickness dermal graft (SDG) applied at the forehead. (A) Early findings of the scalp SSG and SDG at 5 days postoperatively. (B) Excellent outcomes of the grafts at 2 years postoperatively.
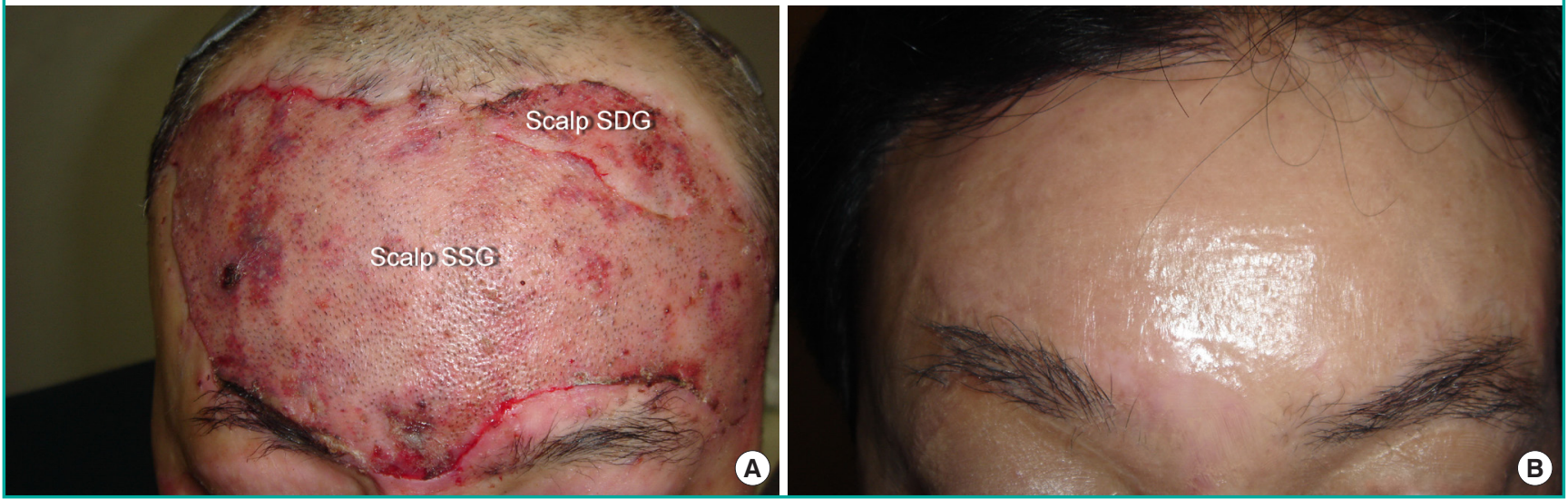


\section{Fig. 7. Scalp SSG and SDG in case 5}

Scar shaving and scalp split-thickness skin graft (SSG) and split-thickness dermal graft (SDG) applied at the left knee. (A) Immediate postoperative appearance. (B) Comparison of the colors of the grafts at 1 year postoperatively.

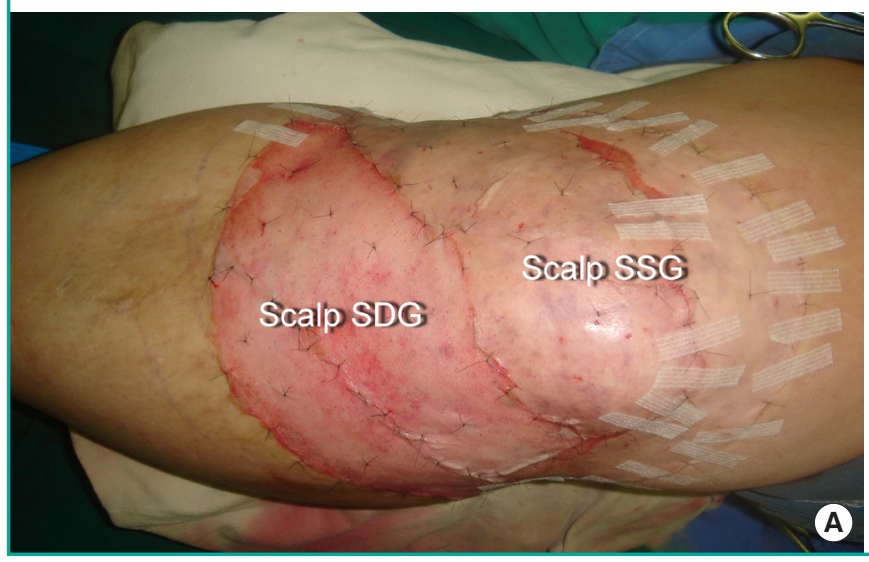

the recipient sites of scalp SDGs were the face $(\mathrm{n}=6)$, lower extremity $(n=6)$, upper extremity $(n=2)$ and body $(n=2)$ (Figs. 5-7).

The mean thickness of scalp SSGs was 0.0089 inches (range, $0.008-0.012$ inches). The mean area of scalp SSGs was 151.8 $\mathrm{cm}^{2}$ (range, $2-266 \mathrm{~cm}^{2}$ ). The mean thickness of scalp SDGs was 0.0089 inches (range, $0.008-0.010$ inches). The mean area of scalp SDGs was $88.2 \mathrm{~cm}^{2}$ (range, $10-200 \mathrm{~cm}^{2}$ ). The mean epithelialization time of scalp SDGs was 10 days (range, 7-14 days). The mean thickness of scalp donor defects was 0.018 inches (range, 0.016-0.022 inches). The mean healing time of scalp donors was 9.9 days (range, 7-14 days).

Partial loss of scalp SSGs occurred in two cases. Partial loss of scalp SDGs occurred in five cases. The recipient's grafts were lost due to infection by multidrug-resistant Acinetobacter baumannii in two cases and methicillin-resistant Staphylococcus aureus (MRSA) in one case. The donor sites of the secondary SSGs for graft loss were the thigh $(n=2)$ and the scalp $(n=1)$. Tufted scar deformity $(2 \times 5 \mathrm{~cm}$ in size) of the scalp donor site due to MRSA was treated with scar revision.

The Vancouver scar scale scores were similar for each graft after a 1-year follow-up of scalp SSGs (mean score of 2.4) and scalp SDGs (mean score of 1.8) in 11 patients. However, the scalp SDGs showed more hypochromic regions than the scalp SSGs.

\section{DISCUSSION}

Previous reports have described thick scalp microdermis grafts for thigh burns [7] and hair-bearing dermal micrografts for hiding cleft lip scars [8]. The double SSG and SDG were compared experimentally for their ability to resurface full thickness skin defects in a pig model [9]. Zakine et al. [6] reported fifteen burn

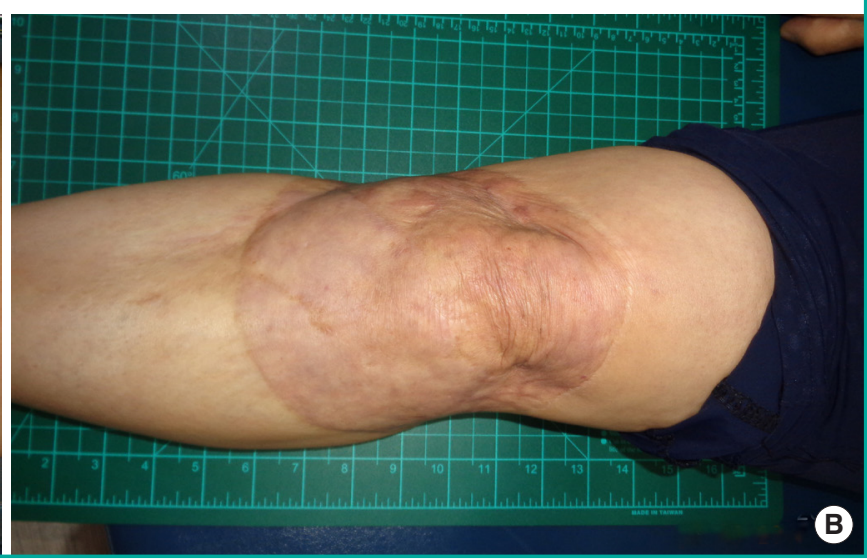

patients treated with two layers of $0.2 \mathrm{~mm} \mathrm{SSG}$, a dermoepidermal graft and a dermal graft, harvested from hairy scalp. Dermal autografts from the scalp have been mainly used in burned wounds, although they can also be used in various other types of wounds. However, the author mainly used scalp SSGs and SDGs for PBSCs and PBSDs of the face or lower extremities. In addition, the mean thickness of scalp SSGs and SDGs was 0.0089 inches $(0.23 \mathrm{~mm})$, and the mean thickness of scalp donor defects was 0.018 inches $(0.46 \mathrm{~mm})$. The mean healing time of the scalp donor site was 9.9 days, similar to that in a previous report of 9.8 days [6].

In wound beds after SSG harvesting, regenerative healing without complications can be induced by maintaining the appropriate thickness of the reticular dermis without damaging the hair follicle. Although stem cells of the hair follicle bulge do not normally contribute to epidermis cells, if the epidermis is damaged, cells from the bulge are recruited into the epidermis and move linearly toward the center of the wound. Bulge-derived cells have epidermal phenotypes but are stem cells involved in acute wound healing, most of which are transiently amplified cells and removed within several weeks $[10,11]$.

Dermal fibroblasts in scalp skin arise from the neural crest in embryonic origin. They undergo differentiation and lineage commitments to give arise to both upper and lower lineages. Dermal papilla, arrector pili muscle, dermal sheath and papillary dermis are derived from the upper lineage [12]. Fibroblasts of dermal papilla, dermal sheath of hair follicle are multipotent stem cells [13]. Epidermal $\beta$-catenin activation stimulates the expansion of the upper dermal lineage in rendering wounds permissive for hair follicle formation [14]. Therefore, the author suggests that scalp donor wounds can be healed early without scar formation by interfollicular regeneration of the dermis and 
Table 2. Comparison of SSG thickness depending on whether the donor skin is from the thigh or scalp

\begin{tabular}{|c|c|c|c|c|c|}
\hline \multirow{2}{*}{ Type of SSG } & \multirow{2}{*}{$\begin{array}{l}\text { Ratio of total skin } \\
\text { thickness }(\%)\end{array}$} & \multicolumn{2}{|c|}{ Thigh skin thickness } & \multicolumn{2}{|c|}{ Scalp skin thickness } \\
\hline & & $1.6(\mathrm{~mm})$ & 0.063 (inch) & $2.4(\mathrm{~mm})$ & 0.095 (inch) \\
\hline Epidermal SSG & & 0.1 & 0.004 & 0.1 & 0.004 \\
\hline Thin SSG & $<25(1 / 4)$ & $<0.4$ & $<0.016$ & $<0.6$ & $<0.024$ \\
\hline Intermediate SSG & $\geq 25 \&<50(2 / 4)$ & $\geq 0.4 \&<0.8$ & $\geq 0.016 \&<0.032$ & $\geq 0.6 \&<1.2$ & $\geq 0.024 \&<0.047$ \\
\hline Thick SSG & $\geq 50 \&<75(3 / 4)$ & $\geq 0.8 \&<1.2$ & $\geq 0.032 \&<0.047$ & $\geq 1.2 \&<1.8$ & $\geq 0.047 \&<0.071$ \\
\hline
\end{tabular}

by epidermis stem cells from hair follicles in the wound bed. Additionally, interfollicular regenerative healing from hair follicles occurred within 2 weeks regardless of the depth of the skin defect.

The scalp dermal grafts do not need to be covered with epidermal grafts, and they become re-epithelialized in the epithelial cells of the follicular wall and abundant adnexa approximately 10 days after grafting. Meshed SDG could be used for easy graft uptake $[15,16]$. However, unmeshed SDG was used in all cases in this study. The time to epithelialization of dermal grafts harvested from skin from other body regions (9-28 days) [15-18] was longer than that of the scalp dermal grafts in this study. Fewer follicular adnexal epithelial cells are contained in dermal grafts of skin from other body regions than in scalp dermal grafts. Thus, epithelialization time seems to be affected by the size of the epithelialized dermal graft from the epidermis around the dermal graft.

The temporo-occipital area can be easily exposed under general anesthesia and can cover the surgical site with partial hair shaving. The thickest region of scalp skin in individuals was the vertex area. The next thickest region was the occipital area. The thinnest region was the parieto-temporal area. The skin thickness of the scalp donor site was measured by preoperative ultrasonography (Fig. 2). The adjusted thickness of the dermatome in harvested skin grafts varies depending on the operator's harvesting techniques and tumescent skin expansion. Therefore, a histological evaluation of the obtained skin thickness is required (Fig. 3). The maximum thickness adjustment of the Zimmer dermatome for harvesting skin is 30 (0.03 inches, $0.76 \mathrm{~mm})$. The skin thickness is $0.05 \mathrm{~mm}$ for the epidermis and $0.1 \mathrm{~mm}$ for the epidermal rete ridge [6]. The SSG type is determined by adjusting the dermatome to an appropriate thickness according to the thickness of the donor skin. SSGs of 0.4 to $<0.8 \mathrm{~mm}$ in thickness are considered intermediate SSGs when obtained from adult thigh donor regions of $1.6 \mathrm{~mm}$ in thickness but are considered thin SSGs when obtained from adult scalp donor regions of $2.4 \mathrm{~mm}$ in thickness (Table 2). The thickness of the harvested SSG and SDG is usually indicated by the setting of the dermatome rather than the actual measurement of the graft. In practice, we have found that the thickness of grafts may vary for the same dermatome setting. These variations are affected by the pressure exerted on the dermatome, the angle of the dermatome, the amount of the tumescent injection, and the harvest site of the scalp.

The scalp donor sites exhibited early folliculitis and scab formation, and conservative treatment was possible except for in one case of tufted scar deformity, which was treated with scar revision. No alopecia was observed on the scalp donor site. The main treatment for folliculitis and scab formation is conservative, followed by rinsing the scalp with povidone solution and topical antiseptic ointment. This process is repeated daily until folliculitis or scab formation is withdrawn.

During the epithelialization period, scalp dermal grafts can become contaminated by resistant bacteria, causing graft failure. Graft failure occurred at three sites of two patients due to MRAB and MRSA in the scalp SSGs and SDGs, and regrafting was required. $\mathrm{ADM}$ grafts were resistant to infection despite graft loss at that time. The maturation period of combined $\mathrm{ADM}$ and SSGs or SDGs is longer than the maturation period of SSGs or SDGs alone. In conclusion, using the scalp as a donor for double-harvested skin grafts can overcome the disadvantages relating to the relatively small area of the scalp. In addition, deeper wounds of the scalp donor site than previously reported acheived scar-free healing.

\section{NOTES}

\section{Conflict of interest}

No potential conflict of interest relevant to this article was reported.

\section{Ethical approval}

The study was approved by the Institutional Review Board of Bestian Seoul Hospital (IRB No. 2018-08-001) and performed in accordance with the principles of the Declaration of Helsinki. 


\section{Patient consent}

The patients provided written informed consent for the publication and the use of their images.

\section{ORCID}

SukJoon Oh https://orcid.org/0000-0001-7793-6198

\section{REFERENCES}

1. Crawford BS. An unusual skin donor site. Br J Plast Surg 1964;17:311-3.

2. Taylor JW, Wilmore DW, Peterson HD, et al. Scalp as a donor site. Am J Surg 1977;133:218-20.

3. Berkowitz RL. Scalp-in search of the perfect donor site. Ann Plast Surg 1981;7:126-7.

4. Kloti J, Pochon JP. Split skin grafts from the scalp. Prog Pediatr Surg 1981;14:111-22.

5. Mimoun M, Chaouat M, Picovski D, et al. The scalp is an advantageous donor site for thin-skin grafts: a report on 945 harvested samples. Plast Reconstr Surg 2006;118:369-73.

6. Zakine G, Mimoun M, Pham J, et al. Reepithelialization from stem cells of hair follicles of dermal graft of the scalp in acute treatment of third-degree burns: first clinical and histologic study. Plast Reconstr Surg 2012;130:42e-50e.

7. Lin TW. An alternative method of skin grafting: the scalp microdermis graft. Burns 1995;21:374-8.

8. Chen YR, Yeow VK. Cleft lip scar camouflage using dermal micrografts. Plast Reconstr Surg 1999;103:1250-3.
9. Rubis BA, Danikas D, Neumeister M, et al. The use of splitthickness dermal grafts to resurface full thickness skin defects. Burns 2002;28:752-9.

10. Ito M, Liu Y, Yang Z, et al. Stem cells in the hair follicle bulge contribute to wound repair but not to homeostasis of the epidermis. Nat Med 2005;11:1351-4.

11. Levy V, Lindon C, Zheng Y, et al. Epidermal stem cells arise from the hair follicle after wounding. FASEB J 2007;21: 1358-66.

12. Driskell RR, Watt FM. Understanding fibroblast heterogeneity in the skin. Trends Cell Biol 2015;25:92-9.

13. Mistriotis P, Andreadis ST. Hair follicle: a novel source of multipotent stem cells for tissue engineering and regenerative medicine. Tissue Eng Part B Rev 2013;19:265-78.

14. Driskell RR, Lichtenberger BM, Hoste E, et al. Distinct fibroblast lineages determine dermal architecture in skin development and repair. Nature 2013;504:277-81.

15. Coruh A, Yontar Y. Application of split-thickness dermal grafts in deep partial- and full-thickness burns: a new source of auto-skin grafting.J Burn Care Res 2012;33:e94-e100.

16. Lindford AJ, Kaartinen IS, Virolainen S, et al. The dermis graft: another autologous option for acute burn wound coverage. Burns 2012;38:274-82.

17. Han SK, Yoon TH, Kim JB, et al. Dermis graft for wound coverage. Plast Reconstr Surg 2007;120:166-72.

18. Tanabe HY, Aoyagi A, Tai Y, et al. Reconstruction for palmar skin defects of the digits and hand using plantar dermal grafting. Plast Reconstr Surg 1998;101:992-8. 